\title{
Prevalência e fatores associados à Insegurança Alimentar e Nutricional em famílias dos municípios do norte de Alagoas, Brasil, 2010
}

\author{
Prevalence and factors associated with food and nutrition \\ insecurity in families in municipalities of the north \\ of the State of Alagoas, Brazil, 2010
}

\author{
Haroldo da Silva Ferreira ${ }^{1}$ \\ Maria Eduarda Di Cavalcanti Alves de Souza ${ }^{1}$ \\ Fabiana Andréa Moura ${ }^{1}$ \\ Bernardo Lessa Horta ${ }^{2}$
}

${ }^{1}$ Faculdade de Nutrição, Universidade Federal de Alagoas. Campus A.C. Simões BR 104 Norte/Km 96.7, Tabuleiro do Martins. 57.072-970 Maceió Alagoas Brasil. haroldo.ufal@gmail.com ${ }^{2}$ Programa de PósGraduação em Epidemiologia, Universidade Federal de Pelotas.
Abstract The scope of this study was to identify the prevalence and factors associated with food insecurity (INSEC) in families of northern Alagoas. A cross-sectional study was conducted with a random sample of 1444 households classified in accordance with the Brazilian INSEC scale into the following categories: mild, moderate or severe. The prevalence ratio calculated by Poisson regression was used to investigate the association of moderate + severe INSEC with the independent variables in both crude and adjusted analysis. The variables that in the crude analysis reached $p<$ 0.2 were included. INSEC was detected in 919 families, of which, $23.3 \%$ and $14.2 \%$ were in the moderate or severe form, respectively. There was a higher proportion of food insecurity in the families with subjects $<18$ years. The variables that remained independently associated to moderate + severe food insecurity were: rooms in the household $\leq 4$, schooling of the household head $\leq 4$ years, drinking water other than mineral, beneficiary of the Bolsa Familia Program, number of residents in the household $>4$, head of household is female and schooling of the housewife $\leq 4$ years. The conclusion was that the families studied showed a high prevalence of INSEC, especially those with individuals $<18$ years.

Key words Food security, Educational status, Socioeconomic factors
Resumo Objetivou-se identificar a prevalênciae os fatores associados à insegurança alimentar (INSAN) em famílias do norte de Alagoas. Para isso, realizou-se estudo transversal com amostra probabilística de 1.444 famílias. A INSAN (leve, moderada ou grave) foi definida segundo a Escala Brasileira de Insegurança Alimentar. Utilizou-se a razão de prevalência $(R P)$, calculada por regressão de Poisson, para avaliar a associação da INSAN moderada + grave com as variáveis independentes, tanto na análise bruta como na ajustada. Nesta, foram incluidas as variáveis que na análise bruta atingiram $p<0,2$. A INSAN foi detectada em 919 famílias, sendo 23,3\% e 14,2\% nas formas moderada e grave, respectivamente. Houve maior proporção de INSAN nas famílias com indivíduos $<18$ anos. As variáveis que permaneceram independentemente associadas à INSAN moderada+grave foram: cômodos no domicílio $\leq$ 4, escolaridade do(a) chefe da familia $\leq 4$ anos, água de beber diferente de mineral, beneficiário do Programa Bolsa Família, número de residentes no domicílio $>4$, chefe da família ser do sexo feminino e escolaridade materna $\leq 4$ anos. Conclui-se que as famílias estudadas apresentam alta prevalência de INSAN, sobretudo aquelas com indivíduos $<18$ anos.

Palavras-chave Segurança alimentar, Escolaridade, Fatores socioeconômicos 
Introdução

A Declaração Universal dos Direitos Humanos reconhece os direitos sociais como necessários para a garantia de um padrão de vida satisfatório, possibilitando o alcance do pleno potencial de crescimento e desenvolvimento humano. O Estado e seus Governos têm o dever de garantir esses direitos a todos os cidadãos, além de assistir adequadamente os mais vulneráveis e assegurar que, em longo prazo, todos possam ter acesso a uma alimentação adequada por seus próprios meios ${ }^{1,2}$. Apesar disto, esse direito vem sendo violado nas mais diferentes esferas de gestão pública no Brasil ${ }^{3}$.

A falta de acesso à alimentação adequada vivenciada por grandes contingentes populacionais em todo o mundo e, em especial, no Brasil, resulta na fome e na má alimentação ${ }^{4,5}$, fenômenos que, no Brasil, são de ampla magnitude e recorrência histórica e fortemente associados à pobreza e iniquidades sociais, bem como às políticas de desenvolvimento, frequentemente comprometidas com a estrutura de produção e consumo de alimentos, adicionando novos riscos e incertezas no contexto dessa problemática ${ }^{6}$.

Em 2004, a partir da II Conferência Nacional sobre Segurança Alimentar e Nutricional, o conceito de Segurança Alimentar e Nutricional ficou definido como "...a realização do direito de todos ao acesso regular e permanente a alimentos de qualidade, em quantidade suficiente, sem comprometer o acesso a outras necessidades essenciais, tendo como base práticas alimentares promotoras de saúde, que respeitem a diversidade cultural e que sejam social, econômica e ambientalmente sustentáveis"7.

A renda tem sido descrita como o determinante mais importante da insegurança alimentar e nutricional e da fome, devendo ser entendida como uma condição associada, mas não exclusiva ${ }^{8}$. Outros fatores que podem estar associados à insegurança alimentar são a deficiência nas condições de saneamento básico e água de qualidade, acesso precário à educação e saúde, concentração de terra, desemprego e outros, que interferem e comprometem diretamente a qualidade de vida da população ${ }^{7}$.

O presente estudo teve por objetivo avaliar a prevalência e os fatores associados à insegurança alimentar e nutricional na população dos municípios que fazem parte do Consórcio de Segurança Alimentar e Desenvolvimento Local do Norte de Alagoas (CONORTE).

\section{Métodos}

\section{Tipo de estudo e planejamento amostral}

O presente estudo transversal avaliou amostra representativa das famílias residentes nos doze municípios integrantes do CONORTE. A variável de interesse utilizada para o cálculo do tamanho amostral foi a insegurança alimentar e nutricional. $\mathrm{Na}$ ausência de estudos específicos nos municípios selecionados, tomou-se por referência a prevalência de 53,6\% encontrada para o Nordeste por ocasião da Pesquisa Nacional por Amostras de Domicílios (PNAD) de 2004․ Assumindo-se uma margem de erro de 2,5\% para um intervalo de confiança de $95 \%$, seria necessário estudar 1500 famílias.

O CONORTE faz parte dos chamados Consórcios de Segurança Alimentar e Desenvolvimento Local (CONSAD), uma estratégia instituída pelo Governo Federal para promover a segurança alimentar da população. Trata-se de é um arranjo territorial institucionalmente formalizado envolvendo um número definido de municípios que se agrupam para desenvolver ações, diagnósticos e projetos de segurança alimentar e nutricional e desenvolvimento local, gerando trabalho e renda. O CONORTE é o único CONSAD implantado em Alagoas, sendo composto por 12 municípios situados no litoral norte do Estado ${ }^{10}$.

Os municípios foram incluídos com probabilidade igual a 1 . Uma vez estabelecido o $n$ amostral, considerou-se a proporção com que cada município contribuía para o total da população da região do CONORTE. Em seguida, aplicou-se a respectiva proporção em relação ao total da amostra (1500 famílias), determinando-se a quantidade de domicílios a serem investigados em cada município. A partir dessa definição, a amostra foi selecionada em três estágios. O primeiro, por sorteio simples, selecionou-se o setor censitário, em seguida, um quarteirão dentro do setor censitário e, finalmente, os domicílios cujas famílias seriam investigadas. Para definição de quantos setores censitários seriam sorteados por município, considerou-se o número total de domicílios a serem sorteados e que cada setor contribuiria com 20 domicílios. No sorteio dos setores censitários levou-se em consideração, adicionalmente, a proporção da população urbana e rural do respectivo município.

Para o sorteio dos setores censitários, utilizaram-se mapas do Instituto Brasileiro de Geografia e Estatística (IBGE). 
Nas áreas urbanas, os quarteirões identificados nos respectivos mapas dos setores censitários sorteados eram numerados e um deles era aleatoriamente selecionado. Em seguida, sorteava-se uma esquina a partir da qual eram visitadas consecutivamente vinte unidades residenciais. Nas áreas rurais, em virtude das peculiaridades geográficas, investigavam-se as primeiras vinte famílias localizadas aleatoriamente pelo conjunto dos entrevistadores.

\section{Coleta de dados}

A coleta dos dados, precedida de treinamento, estudo piloto e teste dos questionários, foi realizada através de visitas domiciliares durante o mês de janeiro de 2010. As informações de interesse foram obtidas por meio de entrevistas tendo como informante as "donas de casa" dos domicílios sorteados.

A equipe de campo foi formada por um coordenador geral, um supervisor, dois antropometristas e treze entrevistadores. Exceto a coordenação, as demais funções foram distribuídas entre alunos de mestrado e de graduação em nutrição.

A variável dependente foi constituída pela situação de segurança alimentar, classificada segundo a Escala Brasileira de Insegurança Alimentar (EBIA $)^{11}$, cujo formulário consta de 15 perguntas fechadas, com respostas positivas ou negativas sobre a experiência da família nos últimos três meses com relação à alimentação, possibilitando, conforme o número de resposta positivas, discriminá-las em estratos que estimam as diversas condições de insegurança alimentar. Cada resposta afirmativa do questionário representa um ponto, sendo a classificação da escala baseada em sua soma, variando de zero a 15 pontos nas residências com indivíduos menores de 18 anos e de 0 a 9 pontos em residências que não possuem membros com idade inferior a 18 anos (no formulário completo, as últimas seis questões se referem a menores de 18 anos). As famílias com presença de menores de 18 anos são classificadas em situação de insegurança alimentar leve quando apresenta de 1 a 5 respostas afirmativas (de 1 a 3 em residências sem menores de 18 anos); moderada quando apresenta de 6 a 10 respostas afirmativas (de 4 a 6 em residências sem menores de 18 anos); e, grave quando apresentam de 11 a 15 respostas afirmativas (de 7 a 9 respostas em residências sem menores de 18 anos $)^{11}$.

O peso do(a) chefe da família e/ou da "dona da casa" foi obtido em balança antropométrica
Marte PP 180, com capacidade para $180 \mathrm{Kg}$ e sensibilidade para $100 \mathrm{~g}$, devidamente aferidas contra peso padrão. Para aferição da estatura utilizou-se estadiômetro alturexata ${ }^{\circledR}$, dotado de fita métrica inextensível com sensibilidade para $0,1 \mathrm{~cm}$ e amplitude de $220 \mathrm{~cm}$. Os indivíduos foram classificados segundo o Índice de Massa Corporal (IMC) de acordo com os pontos de corte propostos pela Organização Mundial de Saúde ${ }^{12}:<18,5 \mathrm{Kg} / \mathrm{m}^{2}$ (baixo peso); $\geq 18,5$ e $<25$ $\mathrm{Kg} / \mathrm{m}^{2}$ (normal); $\geq 25$ e $<30 \mathrm{Kg} / \mathrm{m}^{2}$ (sobrepeso); $\geq 30 \mathrm{Kg} / \mathrm{m}^{2}$ (obesidade).

Neste estudo considerou-se como "chefe da família" o principal responsável pela subsistência dos demais residentes no domicílio, ou seja, a pessoa que possuía a maior parte da renda familiar.

Por meio de questionários estruturados foram obtidas variáveis socioeconômicas, demográficas, ambientais e de saúde. As variáveis demográficas consideradas foram: número de membros da família, cor/raça do(a) chefe da família, sexo do(a) chefe da família, escolaridade do(a) chefe da família, escolaridade materna, chefe da família sem trabalho/renda e usuário do Programa Bolsa Família/outros; as variáveis de saúde avaliadas foram: hipertensão arterial materna, Índice de Massa Corporal do chefe da família e Índice de Massa Corporal materno. As variáveis ambientais estudadas foram: tipo de casa (alvenaria ou diferente de alvenaria), água para beber, presença de vaso sanitário na residência, quantidade de cômodos na residência, destino das fezes e situação do domicilio (urbano ou rural).

\section{Análise estatística}

Foi realizada dupla entrada de dados no software Epi-Info, versão 3.5.1 (CDC, Atlanta, USA), de forma independente Os dois arquivos foram comparados e os erros de digitação corrigidos. O banco de dados assim constituido foi examinado visando a exclusão de outliers (valores extremos e implausíveis).

Primeiramente realizou-se análise descritiva para medir a frequência das variáveis em estudo. Em seguida, comparou-se a prevalência de insegurança alimentar segundo as diferentes categorias das variáveis analisadas. Para conferir maior especificidade aos resultados das análises de associação, considerou-se como desfecho os casos de insegurança moderada ou grave. A associação foi investigada com base na razão de prevalência (RP) e seu intervalo de confiança a 95\%. As associações que obtiveram um $\mathrm{p}<0,2$ na análise 
bruta foram incluídas num modelo de análise multivariável visando controlar para possíveis fatores de confundimento. Tanto na análise bruta quanto na ajustada, utilizou-se regressão de Poisson com ajuste robusto da variância.

Adotou-se o nível crítico de 0,05 para assumir as associações como estatisticamente significantes $(p<0,05)$.

\section{Aspectos éticos}

Este estudo faz parte do projeto Segurança Alimentar e Nutricional da população dos municípios do Norte de Alagoas, aprovado pelo Comitê de Ética em Pesquisa da Universidade Federal de Alagoas.

Por ocasião da visita domiciliar, foi apresentado o termo de consentimento para que o entrevistado, se fosse o caso, assentasse sua concordância em participar da pesquisa. Para isso, todos foram esclarecidos quanto aos procedimentos a serem empregados, à garantia de sigilo das informações prestadas e à possibilidade de se recusar a participar da investigação.

$\mathrm{O}$ trabalho foi realizado em parceria com as prefeituras dos municípios do CONORTE/AL e acompanhado por membros da equipe da Estratégia Saúde da Família (ESF) cuja área de atuação abrangia cada uma das respectivas comunidades sorteadas para estudo.

\section{Resultados}

Foram investigadas 1.444 famílias. Destas, 78,7\% $(\mathrm{n}=1.136)$ tinham menores de 18 anos em seus domicílios. A Tabela 1 caracteriza as variáveis demográficas, socioeconômicas, ambientais e o Índice de Massa Corporal dos chefes das famílias investigadas

A Tabela 1 mostra que o sexo predominante dos chefes das famílias foi o masculino $(72,4 \%)$, os quais tinham, em média, $45,7 \pm 15,8$ anos de idade. A renda per capita média foi de $\mathrm{R} \$ 5,25 \pm$ $5,70 /$ dia, a qual era ligeiramente superior quando o chefe da família era do sexo masculino: $\mathrm{R} \$$ $5,57 \pm 6,19 /$ dia vs. $R \$ 4,25 \pm 3,75 /$ dia $(p=0,071)$.

A maioria dos(as) chefes das famílias $(64,8 \%)$ e das mães $(56,6 \%)$ apresentava baixa escolaridade ( $\leq 4$ anos de estudo). Em relação ao IMC variou de $14,9 \mathrm{Kg} / \mathrm{m}^{2}$ a $48,7 \mathrm{Kg} / \mathrm{m}^{2}$, chamando à atenção a alta proporção de indivíduos com sobrepeso ou obesidade (54,7\%).

O percentual de famílias com mais de quatro pessoas foi de $41,4 \%$. A quantidade média de cômodos existentes nas residências foi de 5,6 \pm 1,9 . Já a quantidade de pessoas por cômodo apresentou uma média de $1,5 \pm 1,0$ e por cômodos para dormir $2,3 \pm 0,8$ pessoas.

De acordo com a EBIA, $63,7 \%$ das famílias apresentavam algum grau de insegurança alimentar. Essa proporção foi ainda maior entre as famílias que possuíam menores de 18 anos quando comparadas àquelas que não tinham indivíduos dessa faixa etária $(65,8 \%$ vs. $55,4 \%$; $\mathrm{RP}=$ 1,18 ; IC $95 \%=1,07$ a 1,33). A situação de insegurança grave acometeu $14,2 \%$ das famílias ( $\mathrm{n}=$ 205) conforme verifica-se no Gráfico 1.

A Tabela 1 também mostra a distribuição da INSAN moderada + grave segundo as diferentes categorias das variáveis independentes estudadas. Verifica-se que apenas as variáveis cor/raça do chefe da família, dona de casa hipertensa, IMC do(a) chefe da família ou da "dona de casa" e a situação do domicílio (rural ou urbano) não se associaram significantemente $(\mathrm{p}<0,05)$ com a presença de insegurança alimentar moderada e grave. Esta última, contudo, foi incluída no modelo de análise ajustada por ter alcançado um valor $(\mathrm{p}=0,147)$ dentro da margem estabelecida para permanência de uma variável no modelo $(\mathrm{p}<0,2)$.

Os resultados da análise multivariável encontram-se expressos na Tabela 2. As variáveis que permaneceram significantemente associadas ( $p$ $<0,05)$ à insegurança alimentar foram: chefe da família ter escolaridade igual ou inferior a quatro anos de estudo, chefe da família ser do sexo feminino, ser beneficiário do Programa Bolsa Família, número de membros no domicílio igual ou superior a quatro, número de cômodos na residência menor ou igual a quatro, deter condições insatisfatórias da água para beber e escolaridade da dona da casa igual ou inferior a quatro anos de estudo. Já o material de construção predominante da casa diferente de alvenaria, inexistência de vaso sanitário no domicílio, chefe da família sem trabalho e/ou renda, destinação das fezes diferente de fossa séptica ou rede de esgoto, bem como a situação de localização do domicilio (urbano/rural) não se associaram com maior risco de INSAN.

\section{Discussão}

Os municípios integrantes do CONORTE são caracterizados por apresentarem médios e baixos Índices de Desenvolvimento Humano Municipal, problemas relacionados a distorções no 
Tabela 1. Distribuição da insegurança alimentar moderada e grave (INSAN), razão de prevalência (RP) e intervalo de confiança de 95\% (IC95\%) segundo variáveis demográficas, socioeconômicas, ambientais e Índice de Massa Corporal (IMC) dos chefes das famílias e "donas de casa". Municípios do norte de Alagoas, 2010.

\begin{tabular}{|c|c|c|c|c|}
\hline Variável & n (\%) & INSAN(\%) & RP (IC95\%) & $\mathrm{p}$ \\
\hline Número de membros na família & & & & $<0,001$ \\
\hline$\leq 4$ & $846(58,6)$ & 31,9 & 1 & \\
\hline$>4$ & $597(41,4)$ & 45,4 & $1,42(1,24 ; 1,62)$ & \\
\hline Cor/raça do (a)chefe da família & & & & 0,258 \\
\hline Branca & $237(16,5)$ & 34,2 & 1 & \\
\hline Não branca & $1.203(83,5)$ & 38,2 & $1,12(0,92 ; 1,35)$ & \\
\hline Sexo do (a) chefe da família & & & & 0,019 \\
\hline Masculino & $1.046(72,4)$ & 35,7 & 1 & \\
\hline Feminino & $398(27,6)$ & 42,2 & $1,18(1,03 ; 1,36)$ & \\
\hline Escolaridade do (a) chefe da família & & & & $<0,001$ \\
\hline$>4$ anos & $450(35,2)$ & 22,4 & 1 & \\
\hline$\leq 4$ anos & $827(64,8)$ & 43,8 & $1,95(1,62 ; 2,35)$ & \\
\hline Escolaridade da dona de casa & & & & $<0,001$ \\
\hline$>4$ anos & $545(43,4)$ & 28,8 & 1 & \\
\hline$\leq 4$ anos & $710(56,6)$ & 42,8 & $1,49(1,27 ; 1,74)$ & \\
\hline Dona de casa hipertensa & & & & 0,665 \\
\hline Não & $814(62,8)$ & 37,1 & 1 & \\
\hline Sim & $483(37,2)$ & 38,3 & $1,03(0,89 ; 1,19)$ & \\
\hline \multicolumn{5}{|l|}{ IMC do chefe da família $\left(\mathrm{Kg} / \mathrm{m}^{2}\right)$} \\
\hline Baixo Peso $(<18,5)$ & $20(2,6)$ & 45,0 & $1,01(0,61 ; 1,68)$ & 0,942 \\
\hline Eutrofia $(18,5$ a 24,9$)$ & $335(42,7)$ & 44,2 & 1 & - \\
\hline Sobrepeso $(25$ a 23,9$)$ & $261(33,2)$ & 43,3 & $0,99(0,82 ; 1,18)$ & 0,829 \\
\hline Obesidade $(>30)$ & $169(21,5)$ & 39,1 & $0,88(0,71 ; 1,10)$ & 0,280 \\
\hline \multicolumn{5}{|l|}{ IMC materno $\left(\mathrm{Kg} / \mathrm{m}^{2}\right)$} \\
\hline Baixo Peso $(<18,5)$ & $30(2,3)$ & 33,3 & $0,93(0,55 ; 1,57)$ & 0,786 \\
\hline Eutrofia $(18,5$ a 24,9$)$ & $455(34,7)$ & 35,8 & 1 & - \\
\hline Sobrepeso $(25$ a 23,9$)$ & $459(35,1)$ & 38,8 & $1,08(0,91 ; 1,28)$ & 0,356 \\
\hline Obesidade $(>30)$ & $365(27,9)$ & 36,7 & $1,02(0,85 ; 1,23)$ & 0,793 \\
\hline Chefe da família sem trabalho / renda & & & & 0,002 \\
\hline Não & $1.286(94,9)$ & 35,9 & 1 & \\
\hline Sim & $69(5,1)$ & 52,2 & $1,45(1,14 ; 1,84)$ & \\
\hline Usuário do Programa Bolsa Família/ outros & & & & $<0,001$ \\
\hline Não & $712(49,5)$ & 27,7 & 1 & \\
\hline Sim & $726(50,5)$ & 47,4 & $1,71(1,49 ; 1,97)$ & \\
\hline Tipo de casa & & & & $<0,001$ \\
\hline Alvenaria & $1.271(89,6)$ & 35,6 & 1 & \\
\hline Diferente de alvenaria & $148(10,4)$ & 54,7 & $1,54(1,31 ; 1,81)$ & \\
\hline Água para beber & & & & $<0,001$ \\
\hline Mineral & $128(8,9)$ & 10,2 & 1 & \\
\hline Outras & $1.314(91,1)$ & 40,2 & $3,96(2,35 ; 6,65)$ & \\
\hline Presença de vaso sanitário na residência & & & & $<0,001$ \\
\hline Sim & $1.235(85,7)$ & 34,6 & 1 & \\
\hline Não & $206(14,3)$ & 54,8 & $1,59(1,37 ; 1,84)$ & \\
\hline Quantidade de cômodos na residência & & & & $<0.001$ \\
\hline$>4$ & $1.099(76,1)$ & 33,6 & 1 & \\
\hline$\leq 4$ & $345(23,9)$ & 49,9 & $1,48(1,30 ; 1,70)$ & \\
\hline Destino das fezes & & & & $<0,001$ \\
\hline Adequado & $1.212(84,4)$ & 34,8 & 1 & \\
\hline Inadequado & $224(15,6)$ & 52,7 & $1,51(1,31 ; 1,75)$ & \\
\hline Situação do domicílio & & & & 0,147 \\
\hline Urbano & $1.180(81,7)$ & 36,6 & 1 & \\
\hline Rural & $264(18,3)$ & 41,3 & $1,13(0,96 ; 1,33)$ & \\
\hline
\end{tabular}

Destino das fezes: Adequado (Rede de esgoto; Fossa séptica); Inadequado (fossa negra, terreno baldio). 


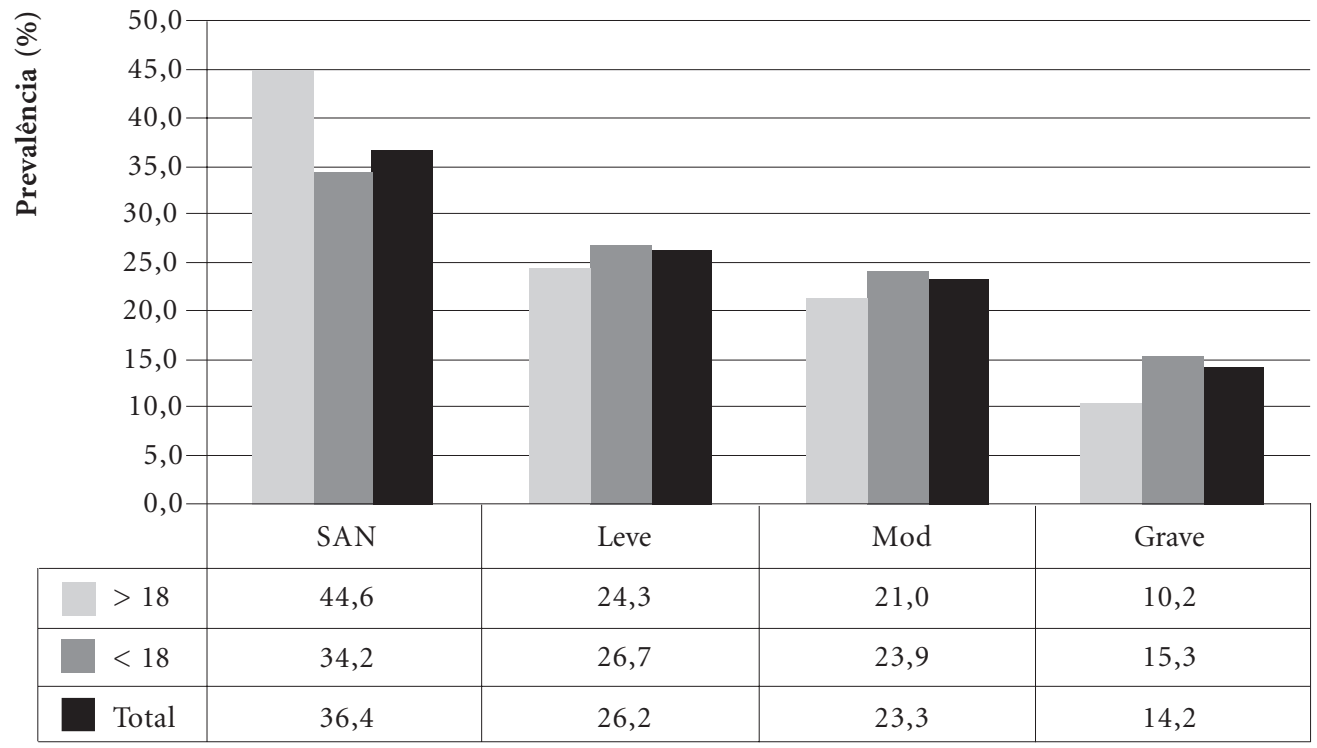

> 18: Famílias que não possuem indivíduos menores de 18 anos no domicílio; < 18: Famílias que possuem indivíduos menores de 18 anos no domicílio; SAN: Diz respeito às famílias classificadas sob a condição de segurança alimentar e nutricional. Leve, Mod e Grave: Diz respeito às famílias classificadas sob a condição de insegurança alimentar e nutricional leve, moderada e grave, respectivamente. A classificação das famílias segundo a condição (in)segurança alimentar e nutricional foi procedida segundo a escala brasileira de segurança alimentar e nutricional ${ }^{12}$.

Gráfico 1. Prevalência das diferentes condições de (In)Segurança Alimentar e Nutricional, segundo a existência ou não de menores de 18 anos nas famílias $(\mathrm{n}=1.444)$ dos municípios do norte de Alagoas, 2010.

Tabela 2. Razão de prevalência e intervalo de confiança de $95 \%$ ajustados por regressão de Poisson para insegurança alimentar moderada e grave em famílias dos municípios do norte de Alagoas, 2010.

\begin{tabular}{lrr}
\hline \multicolumn{1}{c}{ Variável } & RP $($ IC95\%) & p \\
\hline Cômodos no domicílio $\leq 4$ & $1,38(1,16 ; 1,65)$ & $<0,001^{*}$ \\
Escolaridade do(a) chefe da família $\leq 4$ anos & $1,63(1,30 ; 2,05)$ & $<0,001^{*}$ \\
Água de beber diferente de mineral & $2,64(1,47 ; 4,74)$ & $0,001^{*}$ \\
Beneficiário do Programa Bolsa Família & $1,33(1,11 ; 1,59)$ & $0,002^{*}$ \\
Número de residentes no domicílio $>4$ & $1,27(1,08 ; 1,50)$ & $0,004^{*}$ \\
Chefe da família ser do sexo feminino & $1,25(1,06 ; 1,47)$ & $0,006^{*}$ \\
Escolaridade materna $\leq 4$ anos & $1,21(1,01 ; 1,46)$ & $0,042^{*}$ \\
Material de construção predominante da casa diferente de alvenaria & $1,11(0,89 ; 1,40)$ & 0,332 \\
Inexistência de vaso sanitário no domicílio & $1,21(0,77 ; 1,90)$ & 0,409 \\
Chefe da família sem emprego ou renda & $1,11(0,84 ; 1,48)$ & 0,448 \\
Residir em área rural & $0,95(0,76 ; 1,16)$ & 0,594 \\
Destinação das fezes diferente de fossa séptica ou rede de esgoto & $0,95(0,62 ; 1,48)$ & 0,852
\end{tabular}

RP (IC95\%): razão de prevalência e intervalo de confiança de 95\% calculados por regressão de Poisson com ajuste robusto da variância. ${ }^{*}$ Indica significância estatística $(\mathrm{p}<0,05)$ segundo o teste de Wald.

processo de posse e acesso a terras ${ }^{13}$, além de deficiência ou ineficiência de programas de erradicação da pobreza e/ou de geração de emprego e renda ${ }^{14}$. Considerando essas características e as precárias condições socioeconômicas da população da região estudada, não foi surpreendente a elevada proporção de famílias classificadas em situação de insegurança alimentar. 
A prevalência de insegurança alimentar $(63,6 \%)$ na população estudada foi maior do que a encontrada em inquéritos nacionais ${ }^{15,16}$. De acordo com a Pesquisa Nacional de Demografia e Saúde da Criança e da Mulher (PNDS) de 2006, 37,5\% das famílias brasileiras apresentavam algum grau de insegurança alimentar ${ }^{15}$. Já a Pesquisa Nacional por Amostras de Domicílios (PNAD), em $2009^{16}$, revelou números ainda menores para o Brasil (30,2\%). Ainda com base na PNAD-2009, os dados do CONORTE superam as prevalências divulgadas para a Região Nordeste $(46,1 \%)$ e mesmo para o estado de Alagoas (37,1\%).

Prevalências superiores à observada na região do CONORTE foram detectadas por Fávaro et al. ${ }^{17}(75,5 \%)$ e Melo ${ }^{18}$ (90,0\%). Todavia, esses autores estudaram famílias pertencentes a comunidades indígenas, grupamento social reconhecidamente submetido a processos históricos de exclusão social.

Outro fato a ser destacado foi a alta prevalência de insegurança alimentar grave encontrada no norte de Alagoas (14,2\%), ultrapassando em mais do dobro a encontrada para o Brasil pela PNAD-2009 (7,0\%) e superando a prevalência encontrada para o Nordeste $(13,2 \%)$ e para Alagoas $(9,4 \%)^{16}$. Esses dados evidenciam a alta vulnerabilidade social da população residente na região do CONORTE.

$O$ conceito de insegurança alimentar aqui empregado, baseado na EBIA, envolve dimensões tais como a percepção de preocupação e angústia ante a incerteza de dispor regularmente de comida, a utilização de estratégias visando economizar alimento (diluição com redução da qualidade nutritiva, diminuição da diversidade da dieta e da quantidade de alimentos e/ou número de refeições) e, finalmente, a experiência concreta de passar fome por um dia inteiro em virtude de não ter o que comer $^{19}$. Essas dimensões correspondem, respectivamente, aos níveis leve, moderado e grave de insegurança alimentar. Sob tal perspectiva, depreende-se que em cerca de 14,2\% dos domicílios do CONORTE existem moradores passando fome.

No presente estudo, visando imprimir maior especificidade ao indicador de insegurança alimentar, excluiu-se da análise dos fatores associados, a dimensão psicológica, caracterizada pela preocupação de que a comida venha a faltar (insegurança alimentar leve). Assim, foram investigados os fatores associados à insegurança alimentar considerando, de forma conjunta, os níveis moderado e grave, ou seja, aqueles onde assume-se a possibilidade de ocorrência de redução quanti-qualitativa da alimentação da família ${ }^{19}$.
Segundo Fraga et al. ${ }^{20} \mathrm{e}$ Hoffmann ${ }^{21}$, a prevalência de insegurança alimentar é afetada pela presença de indivíduos na família com menos de 18 anos de idade e pelo número de pessoas que residem no domicílio. Assim, tal como também evidenciado no presente estudo, quanto maior o número de menores de 18 anos ou quanto maior o número de moradores no domićlíio, maior a chance da família estar em situação de insegurança alimentar ${ }^{21}$. Tal fato pode estar associado à renda per capita que tende a ser menor nas residências que apresentam menores de idade e nas famílias com maior número de componentes ${ }^{21}$.

Assim como os dados da PNAD-2004 ${ }^{16}$ e de Hoffmann ${ }^{21}$, o fato do chefe da família ser do sexo feminino foi um fator de risco independente para a insegurança alimentar, o que pode ser atribuído ao nível de renda per capita. No presente estudo, os dados mostram que quando a pessoa de referência era do sexo feminino, a renda per capita média era de $\mathrm{R} \$ 4,25 \pm 3,75 / \mathrm{dia}$, ou seja, $R \$ 1,32$ mais baixa do que nas famílias que possuíam o chefe do sexo masculino ( $\mathrm{R} \$ 5,57 \pm$ 6,19/dia). Esses achados confirmam a necessidade de políticas que promovam a equidade entre os gêneros. Vale ressaltar que promover a expansão das oportunidades educacionais e a equidade entre os gêneros está entre as oito metas do milênio estabelecidas pela Organização das Nações Unidas ${ }^{22}$. Para Barroso ${ }^{23}$, o fortalecimento do gênero feminino no sentido do empoderamento, está diretamente relacionado com o aumento do potencial de geração de renda e participação pública além de promover autonomia em questões pessoais, profissionais e também sobre a sua fertilidade. No presente estudo verificou-se que as donas de casa com escolaridade superior a quatro anos de estudo apresentavam uma média de filhos de 3,56 $\pm 2,93 \pm v s .5,55 \pm$ 4,00 filhos das mães com menor escolaridade.

A baixa escolaridade do(a) chefe da família foi outro fator que se associou à insegurança alimentar, corroborando os achados de outros autores $^{24,25}$. A baixa escolaridade da pessoa de referência da residência aumenta a probabilidade da família ter uma alimentação de baixa qualidade, principalmente as crianças, devido à sua influencia sobre o acesso à informação adequada e, na ausência desta, na capacidade de escolha de alimentos saudáveis ${ }^{26}$.

As famílias que residiam em domicílios com um menor número de cômodos apresentaram uma maior prevalência de insegurança alimentar. A média do número de pessoas por cômodo neste estudo foi de $1,50 \pm 1,00$, superior à de 0,87 
$\pm 0,50$ encontrada por Panigassi et al. ${ }^{27}$, segundo os quais, a aglomeração intradomiciliar é uma das variáveis que melhor explicam a insegurança alimentar em seus níveis mais graves.

Neste estudo, as famílias que recebiam os recursos do Programa Bolsa Família apresentaram maior prevalência de insegurança alimentar em comparação às que não tinham esse benefício. Esta observação remete a duas interpretações. A primeira diz respeito à correta focalização do Programa no que concerne ao processo de seleção dos beneficiários, demonstrando que o mesmo está atendendo aos indivíduos que, de fato, encontram-se em maior vulnerabilidade social. Por outro lado, sugere que o Bolsa Família não está sendo capaz de garantir a segurança alimentar e nutricional das famílias. Contudo devido ao caráter transversal deste estudo, esta observação fica comprometida, razão pela qual se sugere estudos específicos para responder a tal questão.

Ainda nessa linha, não existem dúvidas quanto ao fato de que os recursos do Bolsa Família aumentam a capacidade de provimento de alimentos no âmbito familiar ${ }^{28}$. A questão é se esse aumento quantitativo na disponibilidade de alimento é acompanhada do ponto de vista qualitativo. No presente estudo, não se teve esse objetivo, mas um Relatório Técnico do Programa Bolsa Família refere que as famílias em insegurança alimentar, quando passam a receber o valor do benefício, aumentaram o consumo de açúcares, alimentos industrializados, óleos, carnes, feijões, frutas, leites, biscoitos, arroz e cereais, sendo que as maiores proporções são para os alimentos de maior densidade calórica ${ }^{29}$, situação não desejável num momento em que a prevalência de obesidade avança de forma preocupante em todos os seguimentos sociais da população. Neste contexto, convém ressaltar o papel imprescindível dos gestores das políticas públicas de combate à inse- gurança alimentar, considerando que a avaliação do impacto do incremento de renda na alimentação das famílias funciona como subsídio para a elaboração de estratégias para melhorar a qualidade da alimentação dos indivíduos, através da educação alimentar e nutricional ${ }^{27}$.

Outra variável que, neste estudo, se manteve associada à insegurança alimentar após o ajuste para fatores de confundimento foi a origem da água de beber. As famílias cuja água de beber provinha de fontes não segura, tais como cacimba, água de rio e açudes apresentaram maior prevalência de insegurança alimentar. A água potável é um fator ligado diretamente ao estado de saúde do individuo. Ademais, a água de qualidade é um direito humano que deve ser garantido para toda a população $0^{30}$, sendo, portanto, uma prerrogativa para a garantia da segurança alimentar e nutricional e para a saúde e o desenvolvimento econômico local ${ }^{31}$.

É válido enfatizar que o instrumento utilizado no presente estudo para mensuração da insegurança alimentar é subjetivo em sua medida, uma vez que depende da fidedignidade da informação fornecida pelo sujeito entrevistado. Todavia, os resultados obtidos parecem tanto compatíveis com os demais indicadores analisados como coerentes com os estudos de outros autores ${ }^{19,32-34}$.

Diante da alta prevalência de insegurança alimentar encontrada e considerando os problemas de saúde pública decorrentes desta situação, os resultados ora encontrados recomendam a máxima atenção por parte dos gestores das políticas públicas, em suas diferentes esferas, no sentido de promover ações que garantam o direito humano à alimentação adequada a essa população. Para isso, os dados ora apresentados devem ser considerados, pois permitem um melhor planejamento e avaliação das ações instituídas objetivando a reversão do problema. 


\section{Colaboradores}

MECA Souza trabalhou na concepção, coleta e tabulação dos dados e na redação da versão inicial do artigo. HS Ferreira elaborou, coordenou e obteve financiamento para o projeto. FA Moura teve participação na concepção e na redação da versão inicial do artigo. BL Horta colaborou da definição do plano de análise e na revisão do texto final. Todos os autores aprovaram a versão final do artigo.

\section{Referências}

1. Robinson M. The human right to food and nutrition. United Nations High Commissioner For Human Rights. SCN News 1999; (18):17-18.

2. Brasil. Ministério da Saúde (MS). Politica Nacional de Alimentação e Nutrição. Brasília: MS; 2000. [Internet]. [acessado 2010 jan 15]. Disponível em: http:// 200.214.130.94/nutricao/documentos/pnan.pdf

3. Brasil. Conselho Nacional de Segurança Alimentar e Nutricional. A Segurança Alimentar e Nutricional e o Direito Humano à Alimentação Adequada no Brasil. indicadores e monitoramento - da constituição de 1988 aos dias atuais. Brasília; 2010. [Internet]. [acessado 2013 jun 4]. Disponível em: http://www.abrandh.org. $\mathrm{br} /$ UserFiles/File/a-seguranca-alimentar-e-nutricional -e-o-direito-humano-a-alimentacao-adequada-nobrasil(2).pdf

4. Castro J. Geografia da Fome. Rio de Janeiro: O Cruzeiro; 1946.

5. Takagi M, Silva JG, Grossi M. Pobreza e Fome: em busca da metodologia para quantificação do problema no Brasil. Campinas: Universidade Estadual de Campinas; 2001. Texto para discussão.

6. Pessanha LDR. A Experiência brasileira em políticas públicas para a garantia do direito ao alimento. Escola Nacional de Ciências Estatísticas, Departamento de Ensino e Pesquisa. Rio de Janeiro. 2002. [Internet]. [acessado 2013 abr 16]. Disponível em: http:/ /www.ence.ibge.gov.br/c/document_library/get_ file? uuid=a1c57282-e6d5-4561-95b2-5aacdd2312c0 \&groupId $=37690208$

7. Kepple AW, Segall-Corrêa AM. Conceituando e medindo segurança alimentar e nutricional. Cien Saude Colet 2011; 16(1):187-199.

8. Panigassi G. Inquérito populacional sobre a percepção da segurança alimentar intrafamiliar no município de Campinas. 2005. [Internet]. [acessado $2011 \mathrm{fev}$ 1]. Disponível em: http://cutter.unicamp.br/document/?code $=$ vtls000389627

9. Instituto Brasileiro de Geografia e Estatística. Pesquisa Nacional por Amostras em Domicílio - PNAD. 2006. [Internet]. [acessado 2011 jan 27]. Disponível em: http://www.ibge.gov.br/home/estatistica/populacao/ trabalhoerendimento/pnad2004/comentarios2004. pdf

10. Brasil. Ministério do Desenvolvimento Social e Combate à Fome (MDS). Dicionário Social do MDS. [Internet]. [acessado 2011 jan 29]. Disponível em: http://aplicacoes.mds.gov.br/sagi/dicivip/pages/ index.php?paginaAIncluir $=$ conPrograma\&consulta Por=nome

11. Segall-Corrêa AM, Pérez-Escamilla R, Maranha LK, Sampaio MFA, Yuyama L, Alencar F, Vianna RPT, Vieira ACF, Coitinho D, Schmitz BS, Leão MM, Gubert M. Acompanhamento e avaliação da segurança alimentar de famílias brasileiras: validação de metodologia e de instrumento de coleta de informação. Campinas: Universidade Estadual de Campinas, Organização Pan-Americana da Saúde, Ministério de Saúde; 2003. (Relatório Técnico).

12. World Health Organization (WHO). Obesity: preventing and managing the global epidemic. Geneva: WHO; 1998. Report of a WHO Consultation on Obesity. 
13. Instituto Brasileiro de Geografia e Estatística (IBGE). Produção Agrícola Municipal. Rio de Janeiro: IBGE; 2005.

14. Brasil. Ministério do Desenvolvimento Agrário (MDA). Plano territorial de desenvolvimento rural sustentável (PTDRS). Território do Litoral Norte Alagoano. Brasília: MDA; 2006.

15. Brasil. Ministério da Saúde (MS). Pesquisa Nacional de Demografia e Saúde da Criança e da Mulher PNDS. 2008. [Internet]. [acessado 2010 out 7]. Disponível em: http://bvsms.saude.gov.br/bvs/pnds/ img/relatorio_final_pnds2006.pdf

16. Instituto Brasileiro de Geografia e Estatística (IBGE). Pesquisa Nacional por Amostras em Domicílio - PNAD (2009). Rio de Janeiro: IBGE; 2010.

17. Fávaro T, Ribas DLB, Zorzatto JR, Segall-Corrêa AM, Panigassi G. Segurança alimentar em famílias indígenas Teréna, Mato Grosso do Sul, Brasil. Cad Saude Publica 2003; 23(4):785-793.

18. Melo MCO. Situação de (in)segurança alimentar e nutricional em índios Karapotó da aldeia Plak-ô em São Sebastião, Estado de Alagoas [dissertação]. Maceió: Universidade Federal de Alagoas; 2009.

19. Marín-León L, Segal-Corrêa AM, Panigassi G. A percepção de insegurança alimentar em famílias com idosos em Campinas, São Paulo, Brasil. Cad Saude Publica 2005; 21(5):1433-1440.

20. Fraga DS, Camargo M, Vitolo MR, Santos RPM. Relação entre insegurança alimentar e perfil socioeconômico das famílias residentes em uma área de risco de Porto Alegre/RS. Boletim da Saúde 2007: 21(1):67-76.

21. Hoffmann R. Determinantes da insegurança alimentar no Brasil Análise dos Dados da PNAD de 2004. Segurança Alimentar e Nutricional 2008; 15(1):49-61.

22. Organização das Nações Unidas (ONU). Relatório de Desenvolvimento do Milênio. Washington: ONU; 2011.

23. Barroso C. Metas de desenvolvimento do milênio, educação e igualdade de gênero. Cadernos de Pesquisa 2004; 34(123):573-582.

24. Pimentel PG, Sichieri R, Salles-Costa R. Insegurança alimentar, condições socioeconômicas e indicadores antropométricos em crianças da Região Metropolitana do Rio de Janeiro/Brasil. R. Bras. Est. Pop. 2009; 26(2):283-294.

25. Santos JV, Gigante DP, Domingues MR. Prevalência de insegurança alimentar em Pelotas, Rio Grande do Sul, Brasil, e estado nutricional de indivíduos que vivem nessa condição. Cad Saude Publica 2010; 26(1):41-49.
26. Molina MCB, Lopéz PM, Faria CP, Cade NV, Zandonade E. Preditores socioeconômicos da qualidade da alimentação de criança. Rev Saude Publica 2010; 44(5):785-792.

27. Panigassi G, Segall-Corrêa AM, Marin-León L, Pérez-Escamilla R, Sampaio MFA, Maranha LK. Insegurança alimentar como indicador de iniquidade: análise de inquérito populacional. Cad Saude $\mathrm{Pu}$ blica 2008; 24(10):2376-2384.

28. Burlandy L. Transferência condicionada de renda e segurança alimentar e nutricional. Cien Saude Colet 2007; 12(6):1441-1451.

29. Instituto Brasileiro de Análises Sociais e Econômicas (Ibase). Repercussões do Programa Bolsa Família na Segurança Alimentar e Nutricional das famílias beneficiadas. Rio de Janeiro: Ibase; 2008. Relatório Técnico.

30. Brasil. Conselho Nacional de Segurança Alimentar (Consea). O acesso e os usos da água no contexto da soberania e da Segurança Alimentar e Nutricional. Brasília: Consea; 2008.

31. Watkins K. Relatório do Desenvolvimento Humano 2005. Programa das Nações Unidas para o Desenvolvimento (PNUD). Washington: PNUD; 2005.

32. Salles-Costa R, Pereira RA, Vasconcellos MTL, Veiga GV, Marins VMR, Jardim BC. Associação entre fatores socioeconômicos e insegurança alimentar: estudo de base populacional na Região Metropolitana do Rio de Janeiro, Brasil. Rev. Nutr. 2008; 21(Supl.):99s-109s.

33. Vianna RPT, Segall-Corrêa AM. Insegurança alimentar das famílias residentes em municípios do interior do estado da Paraíba, Brasil. Rev. Nutr. 2008; 21(Supl.):111s-122s.

34. Santos LP, Costa MG, Santos JV, Lindemann IL, Gigante DP. Comparacao entre duas escalas de seguranca alimentar. Cien Saude Colet 2014; 19(1):279286.

Artigo apresentado em 05/05/2013

Aprovado em 31/05/2013

Versão final apresentada em 04/06/2013 